\title{
Online Peer Support Groups to Combat Digital Addiction: User Acceptance and Rejection Factors
}

\author{
Manal Aldhayan ${ }^{1}$, Sainabou Cham ${ }^{1}$, Theodoros Kostoulas ${ }^{1}$, Mohamed Basel Al- \\ $\operatorname{mourad}^{2}$, Raian $\mathrm{Ali}^{1}$ \\ ${ }^{1}$ Bournemouth University, UK \\ \{maldhayan, scham, tkostoulas, rali\}@bournemouth.ac.uk \\ ${ }^{2}$ Zayed University, UAE \\ basel.almouradezu.ac.ae
}

\begin{abstract}
The obsessive usage of digital media may exhibit symptoms traditionally associated with behavioural addictions such as mood modification, salience, tolerance and conflict. The educational methods, interventions, and treatments available to prevent or control such a digital addiction are, currently, very limited. Digital Addiction (DA) is yet not formally recognised as a mental disorder by the Diagnostic and Statistical Manual of Mental Disorders. Recently, in 2018, the World Health Organization recognised gaming disorder. Fortunately, the nature of digital media can also help the hosting of methods and mechanics to combat DA, e.g. in the monitoring of online usage and enabling individuals to stay in control of it. One of the techniques proposed in the literature is Online Peer Groups platforms, towards allowing people to form a group and provide peer support to control and regulate their usage, collectively. Online peer support groups are meant to provide peer support, counselling, motivational and learning environment, and ambivalence reduction through sharing and hope installation. However, there is a lack of research about the factors influencing people with DA to accept or reject online peer support groups. In this work, we conduct user studies and explore the acceptance and rejection factors to join and participate in such DA regulation and relapse prevention method. This will help to design and introduce the method and increase its adoption.
\end{abstract}

Keywords: Online peer groups, digital addiction, behavioural change

\section{Introduction}

Digital media such as social networks, gaming and online shopping have become firmly established as part of our daily lives. Such media empowered social connectedness, information exchange and freedom of information exchange. However, despite the benefits, some usages of digital media can be considered compulsive and obsessive leading to negative consequences such as reduced involvement in real-life communities and a lack of sleep [1]. Recent studies have indicated that users who become addicted to digital technologies exhibit the same symptoms as other behavioural addiction such as salience, conflict and mood modification [2, 3]. 
The preventative, control and recovery mechanisms available for Digital Addiction (DA) are currently very limited. One of the reasons for such absence is that, with the exception of online games, DA is still not classified as a mental disorder in the latest 5 th edition of the Diagnostic and Statistical Manual of Mental Disorders (DSM). Most of the existing research on DA focuses on the users' psychology, i.e. their reasons for the overreliance on social media and the relationship with personality traits [4]. Only few works placed software design at the centre of the DA problems, both in facilitating it and also in combatting it, e.g. the digital addiction labels and the requirements engineering for digital well-being requirements in [6,7].

Despite the proliferation of software to assist with behavioural change, there appear to be several issues with the acceptance and efficacy of such application and whether they should be used as a primary or auxiliary mechanism. The perception of their role has changed following some failures and the recognition of associated risks [8]. This calls for further research to establish a deeper understanding of what role such software can play and how their design process would be conducted. A core element of the process for addressing those issues is to capture users' digital wellbeing requirements and to explore acceptance and rejection factors.

Behaviour change theories seek to link the intention to change the behaviour with the act of doing so and are used to predict and promote behavioural change [5]. Such methods have been used in the field of addiction and may provide useful insight into supporting changes in addictive behaviours. Peer groups are one of the approaches utilised to combat addictive behaviours by providing support and helping in the relapse prevention [9]. Peer groups can be constructed when a group of people share similar interests and in view of supporting and influencing each other's behaviour [10]. Alrobai et al. [11] were among the first to examine the peer group approach by utilising social computing techniques themselves to combat DA. Alrobai et al. [13] focused on the processes involved when running the group, e.g. the roles involved in doing so and the steps to be taken to prevent relapse. In that work, the authors only considered the operational phase of a peer support group and did not address the earlier stage of the lifecycle of peer groups, namely, peer's acceptance and agreeable protocols. Overall, there is a need for establishing a deeper understanding of acceptance and rejection factors of such software-assisted method by people with DA. This understanding will inform the strategies used to introduce such software, as well as its configuration and governance processes.

In this paper, we explore the acceptance and rejection factors of online peer support groups as a mechanism for combatting DA. Acceptance is vital as members of the group report their online use, emotions and intentions on a voluntary basis. Although technology can be designed to monitor digital usage, people can always find ways around it if they so desire, e.g. using different devices and accounts or claiming that the use was necessary for work reasons. As a method, we adopted qualitative research employing a secondary analysis of focus groups, originally conducted to explore the best design features of peer groups, and further conducting an interview study with 16 participants who self-declared to have DA, as a primary data collection technique. Our study is also intended to inform the introduction phase of technologyassisted behaviour change solutions to maximise acceptance and adoption. 


\section{Research Method}

We adopted a qualitative method to explore acceptance and rejection factors of people with DA to join online peer support groups for combating their DA. We collected and utilised data from two studies to increase the credibility and coverage of the findings.

In the first study, we performed a secondary analysis of a focus group study of two sessions. The first session aimed at getting insight regarding what the participants think about an online peer group and what they needed to have in it. The second focus group served the purpose of identifying the design features of an online peer group. For this reason, mock design interfaces made based on the result of the first focus group were built and presented. The participants were asked about opinions regarding the mock design. The two focus group sessions were conducted with the same six participants three male and three females, aged between 20 and 26. Participants were selected because they identified themselves as persons who are having a problematic use of social networks. Some of the participants can be considered friends. Though this has some effects in the study, it is, also, beneficial, due to the fact that concerns regarding trust and privacy during the discussion process are suppressed.

The secondary analysis of the data collected from these focus groups was performed using thematic analysis [12]. This analysis revealed the main factors concerning the acceptance of this approach. The findings were used to construct the interview protocol for the primary study and provided a starting template for its analysis. The secondary analysis of the focus group and design session results explored five themes and notable aspects regarding the acceptance and rejection of online peer groups. These five themes related to a) group moderation, b) content, c) governance and operation, d) group coherence and trust and e) goal setting and commitment.

In the second study, an interview study was undertaken in order to elaborate further on these aspects. The interview was conducted with 16 students self-declaring to experience problematic use of online services, e.g., obsessive or compulsive use, 8 males and 8 females, aged between 18 and 35. Each interview lasted between 30 and 40 minutes. The interview questions revolved around the acceptance and rejection factors discovered in the first phase as well as the design features which would support a desired operation of the online peer support group. The interviews were transcribed and analysed via thematic analysis following the theoretical position of Braun and Clarke [12]. The analysis explored different aspects that affect users towards accepting and rejecting an online peer group.

\section{Results}

In this section, we will present the factors which would affect both the acceptance and rejection of people with DA of the online peer support groups. We note here that some of these preferences are contradicted with each other. This would be expected from people with problematic behaviour who often have conflicting requirements about their health, on the one hand, and their desire to continue the problematic behaviour, on the other. Common attitudes and maladaptive behaviours which facilitate that conflict include denial, trivialization and cognitive dissonance [8]. 


\subsection{Online peer support groups to combat DA: acceptance factors}

Different aspects and perceptions explored during the interview contributed to the identification of the factors which affect the user's acceptance to join an online peer group focused on combating digital addiction. These factors should be considered when software and systems engineers design and introduce an online peer group for users who have a problem using digital media. In Fig.1, we illustrate the acceptance factors that affect users regarding their decision in joining an online peer group. In the next subsections, we present these acceptance factors in details.

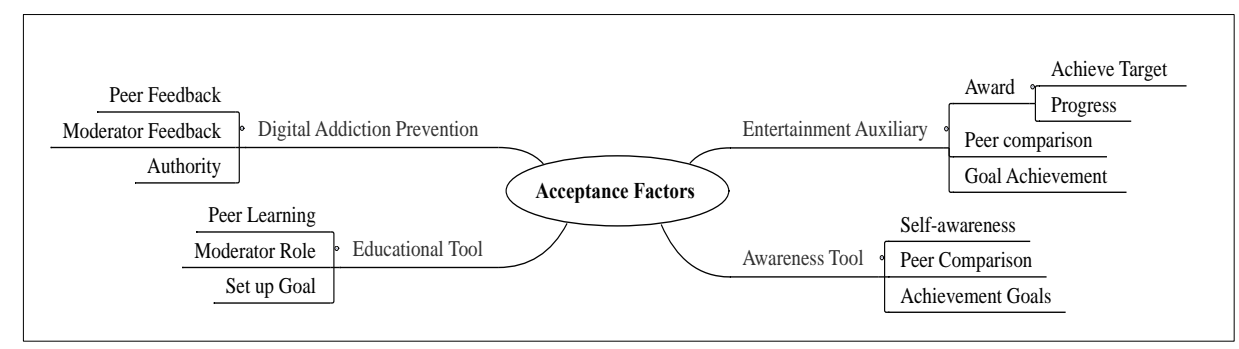

Figure. 1. Online Peer Support Groups to Combat Digital Addiction: Acceptance Factors

Accepting online peer groups as an entertainment auxiliary. An important factor which motivates participants to accept an online peer group is its introduction as one of the "entertainment tools" which will ease the DA prevention and recovery processes. Participants suggested that these tools should include gaming elements which are implemented and adapted as a reinforcement function. This function corresponds to motivating group members to regulate and control digital media usage. That reinforcement function should be designed to be "fun and look like a game" by including "rewards" and "comparison". The participants have three viewpoints in how to establish that: peer comparison, awards and achievement goals.

Peer comparison corresponds to the users comparing their performance and progress with other group members. For example, participants mentioned that comparing usage and progress with group members is "fun". Also, comparing usage would motivate group members to regulate their own digital media usage. It is important to mention that the participants were concerned about comparing their progress with peers that have different levels of addiction since this kind of competition could impact selfesteem and self-efficacy. For example, one participant commented that "when a group has members who have different levels of addiction and skills, and when the system compares progress with them... that might demotivate lower performing people, and then at the end, they might end up relapsing". Overall, the participants agreed that comparing performance and progress with other group members would motivate members to set up goals and targets for the usage and benchmark themselves to others. Additionally, some of the participants stated that they would enjoy sharing and comparing usage with other members and would find it essential and inherent to the sense of being in one group. 
The second viewpoint is that participants prefer the online peer group to provide an award. Specifically, the participants thought that the platform should have a rewards mechanism to "motivate them to achieve group goals" and "to regulate digital media usage". They suggested that the platform could be like a game, with a target to achieve; if a user achieved the target, a reward would be given such as "points or moving from one level to another level". Most of the participants agreed that a reward using a points scheme would be useful and could motivate users to work hard to collect more points. As an example on how these points could be materialized, a participant suggested that a user who collected " 10 points could replace it and get gift or voucher" or could "upgrade their level in the group". Other participants preferred to receive social recognition rewards. For example, members who achieve good progress could have their names or pictures displayed on the main page of the platform or could receive a "congratulations" message from the system, visible to others.

The last viewpoint is that of achieving the group goals. Besides individual and selfset goals, participants would prefer the group to have goals which are established with other group members. In this line, the participants argued that unified goals, applied equally on everyone, or a set of group-agreed goals allocated to members separately would create competition between members that would help them to achieve the goals. They felt that such group goals are "fun by increasing competitiveness between group members", although they argued that unified goals should be "between peers who have similar levels of addiction" and would be more effective with "peers who share the same interests, such as working in the same organization or are postgraduate students".

Accepting online peer groups as a DA awareness tool. Participants appreciated the role of online peer group as an awareness raising and knowledge sharing. Such awareness revolved around self-awareness, peer comparison and ways for the achievement of goals. The first viewpoint corresponds to the expectation of some participants from the online peer group to help them becoming more conscious of their usage and the amount of time spent in "each digital media app". Moreover, they required the online peer group to have a monitoring system, which can track and monitor "the frequency and the time spent using digital media applications" by members. Moreover, the majority of participants commented that they need to be aware of "their level of addiction to digital media usage" because they may be thinking that their usage is "normal, but maybe they are addicted". This conflicts with other statement made by participant around the personal and context-dependent nature of the usage of digital devices and that judgmental approaches towards the claims of having DA are to avoid.

The second important viewpoint about the utilisation of online peer support groups as an awareness tool, related to peer comparisons. In this context, peer comparisons can help group members become aware of their digital media usage and their level of addiction through benchmarking. This can be done through various metrics including time and frequency and also the context in which digital devices are used, e.g. during work and meetings and meals. The comparison can be also non-usage related, e.g. emotions felt while detaching from social media and coping strategies used. Participants agreed that the simple comparison amongst their usages would help increase 
awareness of their usage and if they "used more than the rest, that would motivate them to reduce usage". Also, participants preferred comparing their usage with the group members who have a similar level of addiction, share similar interest, or students enrolled in the same educational programme. A participant, who is a $\mathrm{PhD}$ student, mentioned that "it is useful if the group members are PhD students so when the platform compared my usage with group members and the platform showed my usage is more than others then I have to be more aware of my usage". They prefer that the platform sends a weekly comparison report and that the report compares "their usage with past-usage" and compares "their usage with group members". We note here that metrics for comparison around DA are to be investigated further in future research. Participants emphasised that their use of social media might be for work and hence shall be given a different weighting for its contribution to the problematic usage. They also explained that the calculation of usage shall be more sensitive to the context, e.g. festive season vs. work or sleeping hours.

The third viewpoint is the one of "achieving group goals". The participants prefer to set up group goals which help their commitment to achieving a more in control usage and become aware of their progress towards achieving their targets. Reminders and notification messages seemed to be highly needed as participants stated they might become unconscious of the usage and its amount and context. The group moderator or system could send a notification message to notify the user of the amount of "time spent using digital media" that would help members notice their usage. Furthermore, the system or group moderator could send a warning notification message which "makes the user aware of usage" and when the user "exceeds the time limit of usage". Moreover, the group moderator "would block the digital media apps for a day because the user exceeded the limits". However, it is important to note that the participants were concerned about the "notification time" and "how many messages to send a day", in the sense that they are not in favour of the system exceeding the notification messages more than once a day. We note here again the conflict between being looked after by the system and the requirements of privacy and non-obtrusiveness.

Accepting online peer support groups as an educational tool. The participants considered online peer groups as an educational platform. They generally preferred that such platforms provide functionalities that would help them learn how to control their digital media usage and find life alternatives. They have three viewpoints regarding where to obtain this knowledge from; from peers, group moderators, and by setting up group goals and learn how to achieve them. Regarding the first viewpoint, the participants mentioned that they can learn from peers who had successfully achieved the group goals before by "asking them questions \& receive advice regarding how they reduce usage". Also, peers can learn from each other and "share strategies they follow" to help them control digital media usage while they are all trying in the same time as this can have both educational and motivational value. Moreover, participants mentioned that they can learn from peers' personal and real-world stories thus they prefer interacting with any member who is an ex-addict or one that has successfully achieved the group goals. Such share adds to the relatedness and sense of belonging in the group and acts as a hope installation mechanism. Gaming addiction would be one 
clear example here as participants who used to play games heavily found it difficult to find alternatives to games especially after building their online community around it.

The second viewpoint that affects acceptance of an online peer support group as an educational tool is the moderator's role. The group's moderator has been seen as an educational one and it is expected that the moderator has knowledge and experience in DA. In this sense, the moderator would deliver this "knowledge to the group's members by providing advice". To empower this educational role, the group moderator should be enabled "monitor the group member's usage" and, based on the monitoring result, would then be able to "know their level of addiction and provide support and guidelines suit to them on how to reduce usage". The participants mentioned that they could learn from the moderator's "advice and guidance" which would help them control and combat addiction. Moreover, the participants believed that the online peer group could use some kinds of "role-playing" which is similar to "game learning" [14] as a way for changing behaviour. They suggested that the moderator's role could "rotate", meaning that after a period that "any member who has accumulated high points" could be a group moderator for a period of time. The moderator "could provide advice and rewards to the members" and "set up the group goals".

The last viewpoint around education corresponded to setting up usage goals and learn how to achieve them. Goals seem to have the added value of being an additional motivation to learn. Participants agreed that setting up achievable and realistic goals is also an important factor to sustain the motivation to learn how to achieve them. Despite the fact that some of the participants preferred to set up their own goals, they also mentioned that the group moderator should be able to check if the goals are reasonable and achievable and, in case they are not, the moderator should "explain how they can set up achievable and reasonable goals". In other words, the education can be also around goal setting skills.

Accepting online peer support groups as a digital addiction prevention tool. Using online peer support groups as a mechanism for the prevention of digital addiction seems to be one of the acceptance factors. The participants agreed that such platforms should have monitoring and feedback features administered by the group moderator, peers or the system automatically. In this line, the participants mentioned that "feedback is an important tool for prevention digital media". This feedback can be based on monitoring performance and adherence to the set goals. The participants accepted that a moderator should have the authority and ability to access groupmembers' digital usage and enact precautionary measures. Possibly, members would accept that this access is only from the moment of appointing the moderator, i.e. in the case of rotation based assignment policy. The moderator is expected to observe group members' performance and progress, and, based on that, make a judgment and send feedback and advice to the corresponding member. Also, the moderator should have the authority to take corrective measures if any member does not adhere to the group goals, e.g. "lock some digital media application". Moreover, it is acceptable for a group moderator to observe whether the group members achieve their goals and, if any member struggles to achieve the goals, the moderator is expected to provide supportive information or "amend the goals". Such amendments can be done through dialogue with users or by analysing their performance and profile. 
With regards to how the moderator handles and makes use of the access to the digital usage of the group members, the moderator was expected to (a) make a judgment and (b) send feedback and advice on how to deal with addiction or guidance regarding the member's performance. Some participants preferred the feedback to be "strict, formal and in order" and were in favour of a "warning message" if they exceeded the usage limit or use in an inadequate context, e.g. during work hours. Moreover, participants mentioned that they preferred "moderator feedback to be positive", such as "Congratulations, but you'll need to improve on this and that". Same participants had the two different preferences while others were clear in their specification. This suggests the importance of personality and contextual information around the feedback tone and timing.

\subsection{Online peer support groups to combat DA: rejection factors}

In this section, we will present factors that would lead the users with DA towards rejecting an online peer support group. Figure 2 presents a summary of the rejection factors. These factors are detailed in the following paragraphs.

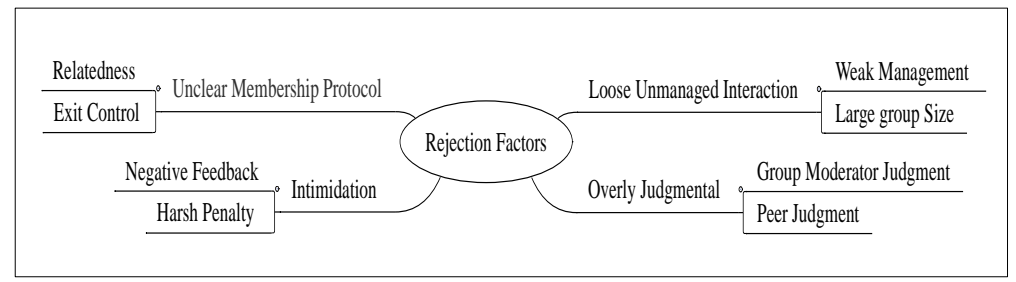

Fig. 2. Online Peer Support Groups to Combat Digital Addiction: Rejection Factors

Rejecting online peer support groups when seen as an intimidation tool. Intimidation is one of the essential reasons for rejecting an online peer group platform. Participants have two viewpoints regarding the rejection due to this reason; the harsh penalty and the negative feedback. Penalties as anticipated by the participants included "blocking from the group" and "writing member name in the main page of the platform as a loser" even in a playful and gameful format. The harsh penalty seems to be affecting members "self-esteem or make member leave the group". Some participants mentioned that harsh penalties mean a "threat" to them and that this would affect their motivation to participate, truthfully, with the group and could lead to leaving the group or adopting workarounds such as using a secret device to access social media in a way that is not monitored by the online peer group software. They commented that "members join the group because they would like to control their digital usage, so they do not prefer to have a penalty" as this seems to be against the free spirit of membership and joining process. A participant stated that she "would not accept a harsh penalty even when I do not achieve the group goals". This again shows the delicacy of implementing rewards and penalty for problematic behaviours and the conflict between users' preferences which necessitates a process of consensus and commitment building when configuring the online per groups platforms and specifying their interaction and governance protocols. 
Participants were much concerned regarding negative feedback messages received from group moderator or peers even if the feedback is factual such as "you compare less favourably to other peers". They prefer motivational feedback and reject the reception of a negative one. A participant commented that "some group members could not achieve the group goals for different reasons such as setting difficult goals or simply could not control usage", and that the feedback from a moderator or peers "should not be harm" additional to what they have already felt and should not "underestimate the user". Overall, participants mentioned that harsh feedback could affect their feeling of membership and relatedness and affect their self-esteem and this could lead them to reject the group. They would prefer to receive a message that motivates and reinforces them to reduce their problem. Having critical feedback is different from negative feedback, and it seems it is a matter of framing and language problem here. Participants did not like the highly serious feedback as although they view DA as a real problem; they reject a framing it as a formal addiction; "I do not like to receive a message says that using digital media for a long-time lead to a mental health problem such as depression". Besides building census and commitment, denial and trivialisation of issues are common attitudes of people with problematic behaviour and need to be dealt with when configuration and starting online peer groups.

Rejecting online peer support groups when seen as overly judgmental. Participants tend to refuse to be judged by others in the group, especially when the judgment is coming from a peer and automated software. The situation can lead even to a harder reaction when peers know each other in person as the judgment will expand to the real personality. A participant mentioned that "if the group members are my friends maybe if I meet them, they will judge me through my digital media usage". Also, participants did not prefer receiving feedback in the form of questions such as "why you are always on Facebook". The main reason for the participants to reject online peer group is that they all have different usage styles and intentions of use when it comes to social media. This observation is an important parameter, which appears that software and peers can not simply judge it. Although less of a concern, participants also had issues by being overly judged by the group moderator. While they tended to accept feedback from the moderator in general, they preferred that the feedback shall include advice or guidance regarding their usage, rather than pure judgment messages. For example, a participant commented that "sometimes the group goals are too hard and I could not achieve the goal", and in this sense, moderators should send feedback to support them and show them strategies rather than sending scores and judgements.

Rejecting online peer supports group when hosting unmanaged interactions. Participants prefer the group interaction to be run and overseen by the group moderator. They had two viewpoints to reject a group in that regard; (a) if it has weak management and (b) if it is a large size so management is hard. Participants would reject a group with a weak moderator who cannot make a decision, such as banning members who are not adhering to the group norms, e.g. in the conversations, and sending warning messages. Participants prefer a group moderator who is "able to control the group connections and oversee messages sent by members". There were concerned about weak management that is unable to stop members annoying others by sending 
feedback against the "group aims" such as "friendship requests", or "jokes". The participants preferred the moderator shall be able to delete any message that does not follow the group aim and send a "warning message". A participant mentioned: "I joined the group because I would like to control my digital media usage and I do not want to receive any friend request from the group members" and the group moderator "has to warn any member who sends a friend request".

The other reason to reject an online peer group is its large size. The participants expressed concerns regarding group size and feared that its management is "difficult" if it is a large one. Specifically, some participants preferred the group size to be small, i.e. from five to ten members. They argued that a big group would be "massive and do not help to achieve the group goals". Furthermore, a large group would be "annoying and would receive many messages", meaning that members could not focus on the group goals. Also, they argued that, in a large group, it "is not easy to track all members and the competition in a small group would make more sense".

Rejecting online peer groups due to unclear membership protocol. Participants expressed concerns regarding (a) relatedness of group members and (b) conditions to exit from the group. Some of the participants rejected to join a group whose group members are friends or relatives. Some of the participants prefer to join a group with people who do not know each other's as that would "make members feel more comfortable and confident" and they would then accept to "share usage, comments, and feedback with peer" because they know "no one would judge [them] in real life". Other participants preferred to join a group of people known to each other's, but they prefer to be "semi-anonymous" and reject to provide their "real name and picture" when it comes to monitoring usages. This can be solved through messages like: one of your friends is having difficulty with games today, what do you like to tell them? Participants agreed that a group of friends "make them trust the group and they will not worry about privacy" as they already know that their usage is problematic. It can be noted here that participants had a paradox between trust and privacy here.

Participants rejected that the group can have conditions or regulation regarding the exit from the group because that would affect "members feeling" and that "they will feel stress and that the group control their freedom". Participants generally agreed that they should be free to leave the group whenever they like, but they would not approve a "member leaving the group without giving notice and explanation why they decide to leave the group". They suggested that when somebody leaves a group, they should invite somebody else to join, especially when the "group size is small" and when leaving may adversely "affect the morale of members to achieve the group aims". Again, we can note the conflicting preferences requiring a resolution process.

\section{Conclusion and Future Work}

In this paper, we explored the factors which affect the acceptance and rejections of people with DA regarding online peer support groups for combating their DA. A range of factors seems to be conflicting. For example, while people like the group to provide a friendly environment where game elements are used, e.g. regarding challenging each other regarding reduction time, they like not to be monitored and judged. 
Similarly, while they appreciate the freedom to join and to leave, they were concerns that this may affect their members and lead to trivialising the process. These observations call for methods to reconcile amongst these requirements. Although participants emphasised a wide range of factors around DA and its judgement, e.g. context and purpose of usage and non-usage related factors such as emotion and preoccupation, their examples tended always to be around time and frequency of using digital devices. This would be expected due to the recent nature of the DA concept and the debate around it. Our future work will look at building a consensus process so that group members can configure agreeable settings of their online platform utilised for peer support groups.

Acknowledgement. This work has been partially supported by the EROGamb project funded jointly by GambleAware and Bournemouth University, SSCoDA project funded by Zayed University and H2020-MSCA-RISE-2017 project, under grant agreement No. 778228 (IDEAL-CITIES).

\section{$5 \quad$ References}

1. Hampton K, Goulet LS, Rainie L, Purcell K.: Social networking sites and our lives. Pew Internet \& American Life Project 16, 1-85 (2011).

2. Griffiths, M.: A 'components' model of addiction within a biopsychosocial framework. Journal of Substance use10 (4), 191-197 (2005).

3. Widyanto, L. and Griffiths, M.: Internet addiction: a critical review. International Journal of Mental Health and Addiction, 4(1), 31-51(2006).

4. Winkler, A., Dörsing, B., Rief, W., Shen, Y. and Glombiewski, J.A.: Treatment of internet addiction: a meta-analysis. Clinical psychology review 33(2), 317-329 (2013).

5. Webb, T.L., Sniehotta, F.F., Michie, S.: Using theories of behaviour change to inform interventions for addictive behaviours. Addiction. 105, 1879-1892 (2010).

6. Ali, R., Jiang, N., Phalp, K., Muir, S., McAlaney, J.: The Emerging Requirement for Digital Addiction Labels. REFSQ. 9013, 198-213 (2015).

7. Alrobai, A., Phalp, K., Ali, R.: Digital Addiction: A Requirements Engineering Perspective. Requirements Engineering: Foundation for Software Quality. 8396, 112-118 (2014)

8. Alrobai, A., McAlaney, J., Phalp, K. and Ali, R. Exploring the risk factors of interactive e-health interventions for digital addiction. International Journal of Sociotechnology and Knowledge Development 8 (2), 1-15(2016).

9. Davidson, L., Chinman, M., Kloos, B., Weingarten, R., Stayner, D., Tebes, J.K.: Peer support among individuals with severe mental illness: a review of the evidence. Clinical Psychology: Science and Practice. 6, 165-187 (2006)

10. Alrobai, A., McAlaney, J., Phalp, K. and Ali, R.: Online peer groups as a persuasive tool to combat digital addiction. In International Conference on Persuasive Technology, 288-300(2016).

11. Alrobai, A., Dogan, H., Phalp, K. and Ali, R.: Building Online Platforms for Peer Support Groups as a Persuasive Behavior Change Technique. In International Conference on Persuasive Technology, 7083 (2018).

12. Braun, V., Clarke, V. and Terry, G.: Thematic analysis. Qual Res Clin Health Psychol, 24, 95-114 (2014).

13. Alrobai, A.: Engineering social networking to combat digital addiction: the case of online peer groups. Doctoral dissertation, Bournemouth University, (2018).

14. Sousa, M.J., Rocha, Á., Game based learning contexts for soft skills development. In World Conference on Information Systems and Technologies, 931-940 (2017). 